\title{
Coordination within the remote sensing payload on the Solar Orbiter mission
}

\author{
F. Auchère ${ }^{1}$, V. Andretta ${ }^{13}$, E. Antonucci ${ }^{2}$, N. Bach ${ }^{12}$, M. Battaglia ${ }^{5}$, A. Bemporad ${ }^{2}$, D. Berghmans ${ }^{9}$, E. Buchlin ${ }^{1}$,
} S. Caminade ${ }^{1}$, M. Carlsson ${ }^{25}$, J. Carlyle ${ }^{6}$, J. J. Cerullo ${ }^{31}$, P. C. Chamberlin ${ }^{11}$, R.C. Colaninno ${ }^{4}$, J. M. Davila ${ }^{11}$, A. De Groof ${ }^{12}$, L. Etesi ${ }^{5}$, S. Fahmy ${ }^{6}$, S. Fineschi ${ }^{2}$, A. Fludra ${ }^{3}$, H. R. Gilbert ${ }^{11}$, A. Giunta ${ }^{3}$, T. Grundy ${ }^{3}$, M. Haberreiter ${ }^{22}$, L. K. Harra ${ }^{22,32}$, D. M. Hassler ${ }^{10}$, J. Hirzberger ${ }^{8}$, R. A. Howard ${ }^{4}$, G. Hurford ${ }^{14}$, L. Kleint ${ }^{5}$,

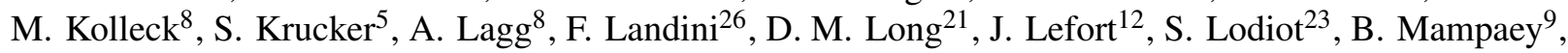
S. Maloney ${ }^{30}$, F. Marliani ${ }^{6}$, V. Martinez-Pillet ${ }^{27}$, D. R. McMullin ${ }^{17}$, D. Müller ${ }^{6}$, G. Nicolini' ${ }^{2}$, D. Orozco Suarez ${ }^{18}$,

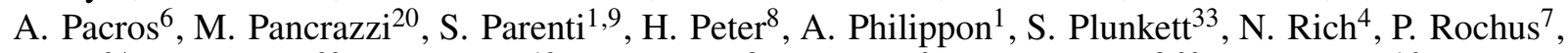
A. Rouillard ${ }^{24}$, M. Romoli ${ }^{20}$, L. Sanchez ${ }^{12}$, U. Schühle ${ }^{8}$, S. Sidher ${ }^{3}$, S. K. Solanki ${ }^{8,29}$, D. Spadaro ${ }^{16}$, O. C. St Cyr ${ }^{11}$, T. Straus ${ }^{13}$, I. Tanco ${ }^{23}$, L. Teriaca ${ }^{8}$, W. T. Thompson ${ }^{19}$, J. C. del Toro Iniesta ${ }^{18}$, C. Verbeeck ${ }^{9}$, A. Vourlidas ${ }^{15}$, C. Watson ${ }^{12}$, T. Wiegelmann ${ }^{8}$, D. Williams ${ }^{12}$, J. Woch ${ }^{8}$, A. N. Zhukov ${ }^{9,28}$, and I. Zouganelis ${ }^{12}$

(Affiliations can be found after the references)

Received 31 October 2019 / Accepted 22 January 2020

\begin{abstract}
Context. To meet the scientific objectives of the mission, the Solar Orbiter spacecraft carries a suite of in-situ (IS) and remote sensing (RS) instruments designed for joint operations with inter-instrument communication capabilities. Indeed, previous missions have shown that the Sun (imaged by the RS instruments) and the heliosphere (mainly sampled by the IS instruments) should be considered as an integrated system rather than separate entities. Many of the advances expected from Solar Orbiter rely on this synergistic approach between IS and RS measurements.

Aims. Many aspects of hardware development, integration, testing, and operations are common to two or more RS instruments. In this paper, we describe the coordination effort initiated from the early mission phases by the Remote Sensing Working Group. We review the scientific goals and challenges, and give an overview of the technical solutions devised to successfully operate these instruments together.

Methods. A major constraint for the RS instruments is the limited telemetry (TM) bandwidth of the Solar Orbiter deep-space mission compared to missions in Earth orbit. Hence, many of the strategies developed to maximise the scientific return from these instruments revolve around the optimisation of TM usage, relying for example on onboard autonomy for data processing, compression, and selection for downlink. The planning process itself has been optimised to alleviate the dynamic nature of the targets, and an inter-instrument communication scheme has been implemented which can be used to autonomously alter the observing modes. We also outline the plans for in-flight cross-calibration, which will be essential to the joint data reduction and analysis.

Results. The RS instrument package on Solar Orbiter will carry out comprehensive measurements from the solar interior to the inner heliosphere. Thanks to the close coordination between the instrument teams and the European Space Agency, several challenges specific to the RS suite were identified and addressed in a timely manner.
\end{abstract}

Key words. space vehicles: instruments - Sun: general - instrumentation: polarimeters - instrumentation: spectrographs - telescopes

\section{The remote sensing challenge}

Due to its comprehensive payload and unique mission profile, Solar Orbiter is very different from previous solar and heliospheric missions. The top-level scientific objectives of the mission (Müller et al. 2013; Müller et al. 2020) require close interaction between the different instruments. This has motivated the formation of the Remote Sensing Working Group (RSWG) and the in-situ Working Group (Walsh et al. 2020) in charge of coordinating the science operations of the remote sensing (RS) and in-situ (IS) instruments, respectively. Initially, the two working groups were mostly devoted to hardware and spacecraft (S/C) interface developments, topics that justified the distinction between RS and IS instruments. As the hardware development progressed, the two groups worked on joint operations within the activities of the Science Operations Working Group (SOWG).

The Solar Orbiter mission profile poses several challenges for the RS instruments. As a deep space mission, the teleme- try (TM) rate varies greatly along a given orbit and from orbit to orbit (Müller et al. 2020), and as a result it is highly constrained compared to recent missions in Earth orbit, such as for example Hinode (Kosugi et al. 2007) and the Solar Dynamics Observatory (SDO, Pesnell et al. 2012). While this is a constraint for the full Solar Orbiter payload, it is felt more strongly by the RS instruments since they are inherently capable of producing orders of magnitude more data than can be transferred to the ground. As a mitigation, the RS science operations have been restricted - from the early mission studies - to three ten-day Remote Sensing Windows (RSW) per six-month orbit, centred on the a priori most interesting vantage points: the perihelion and the two extreme solar latitudes. As a result, the RS suite will not continuously observe the solar activity as has been the case for previous missions. However, when possible, these nominal periods of operation will be complemented by continuous low-cadence, synoptic type observations outside the RSWs (see Sect. 4) providing contextual information to the 
IS payload and other observatories (e.g. Parker Solar Probe, Fox et al. 2016).

Because each orbit is different, the planning concept includes a selection of scientific objectives to be addressed by the payload during the next orbit, typically six months before the start of each six-month planning period. Thus, the high-level science planning for each instrument must be finalised several months before the actual observations. The detailed commanding is frozen two weeks before observations, with modifications only possible a few days before if they are resource-neutral (e.g. TM, power) for $\mathrm{S} / \mathrm{C}$ safety reasons. Combined with the limited duration of the RSWs and limited TM, this was identified very early on as a major hurdle for the observation of intermittent events, such as prominence eruptions, coronal mass ejections (CMEs), and flares. Section 2.2 discusses the strategies devised to overcome these difficulties.

The main characteristics of the six RS instruments are summarised in Table 1. Details can be found in the respective instrument papers given as references. The RS instruments fields of view (FOVs) are illustrated in Fig. 1 for four cases. The $\mathrm{S} / \mathrm{C}$ is nominally pointed at Sun center (Figs. 1a,c, and d), pendicular to the orbital plane. The SPectral Imaging of the Coronal Environment (SPICE) and the high-resolution channels and Helioseismic Imager (PHI) will be able to observe nonSun-centered targets by off-pointing the S/C (Fig. 1b) within the range allowed by the Metis coronagraph safety constraint (Sect. 2.3 and Antonucci et al. 2020). The S/C boresight can be pointed anywhere on the solar disc, for example towards the solar limb (which allows EUI/HRI 174, EUI/HRI $\mathrm{Ly}_{\mathrm{L} \alpha}$, and SPICE to perform limited off-limb observations). As detailed in Sect. 2.3, limb-pointing is not compatible with Metis observations at close Sun distances. Unlike previous solar physics missions, the distance to the Sun will vary by about a factor of two between perihelion and high-latitude windows (Figs. 1a and b). Combined with the changing solar latitude (e.g. Figs. 1a and d), this will result in a variety of geometric configurations. The different scientific objectives will be prioritised accordingly.

The top-level scientific objectives are translated into different operational profiles for the various instruments. For example, at closest approach, EUI and PHI will provide very-high-resolution images ( $0.28^{\prime \prime}$ equivalent from Earth) and are capable of observing at high cadence (down to sub-second). To reduce their downlink volume, these instruments are capable of data selection and extensive onboard processing, by including internal memory equivalent to or larger than their TM orbit allocation. On the other hand, SPICE will perform little onboard data selection or processing, but flexibility of operation comes from the large parameter space offered by it being a spectrograph. Conversely, the Spectrometer/Telescope for Imaging X-rays (STIX) and the Solar orbiter Heliospheric Imager (SoloHI) have been designed to mostly provide observations of synoptic type.

\section{Remote sensing windows and planning}

\subsection{Planning strategy}

As most Solar Orbiter objectives require consistent data sets across the full science payload, the instrument observations must be planned in a coordinated way. This is achieved through the concept of Solar Orbiter Observing Plans (SOOPs, see Zouganelis et al. 2020). These are coordinated campaigns of the nominal roll angle defined by the S/C Z-axis being perof the Extreme Ultraviolet Imager (EUI) and the Polarimetric

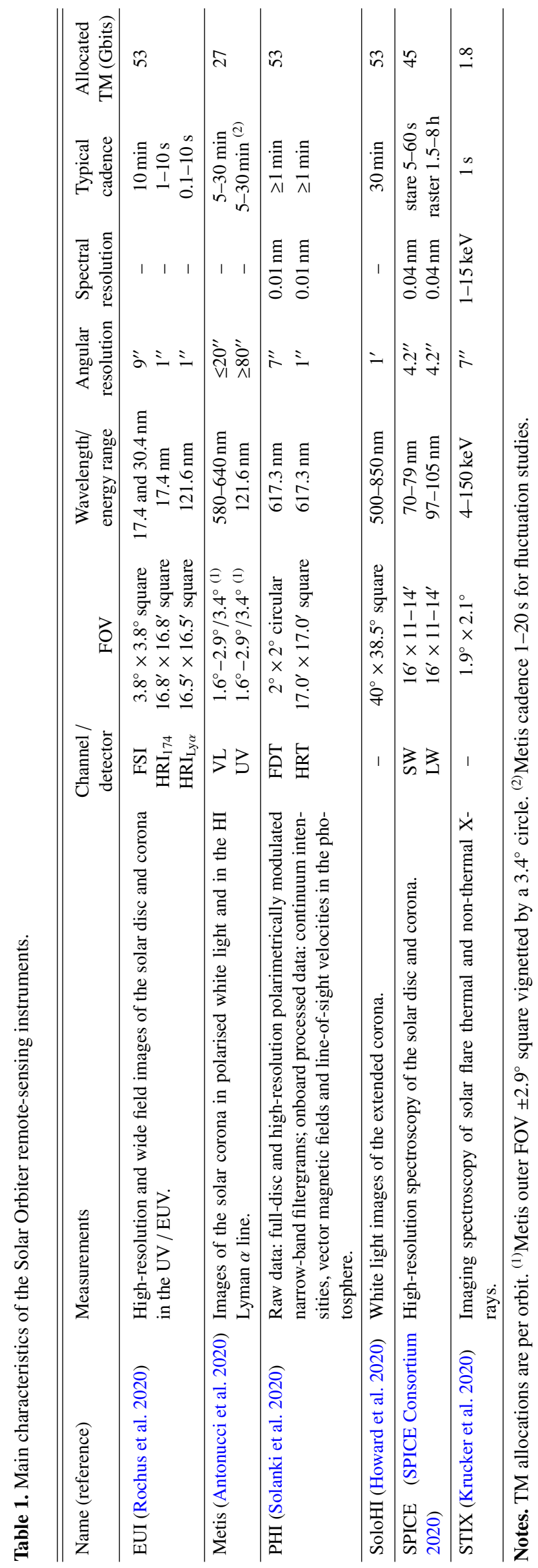

A6, page 2 of 12 

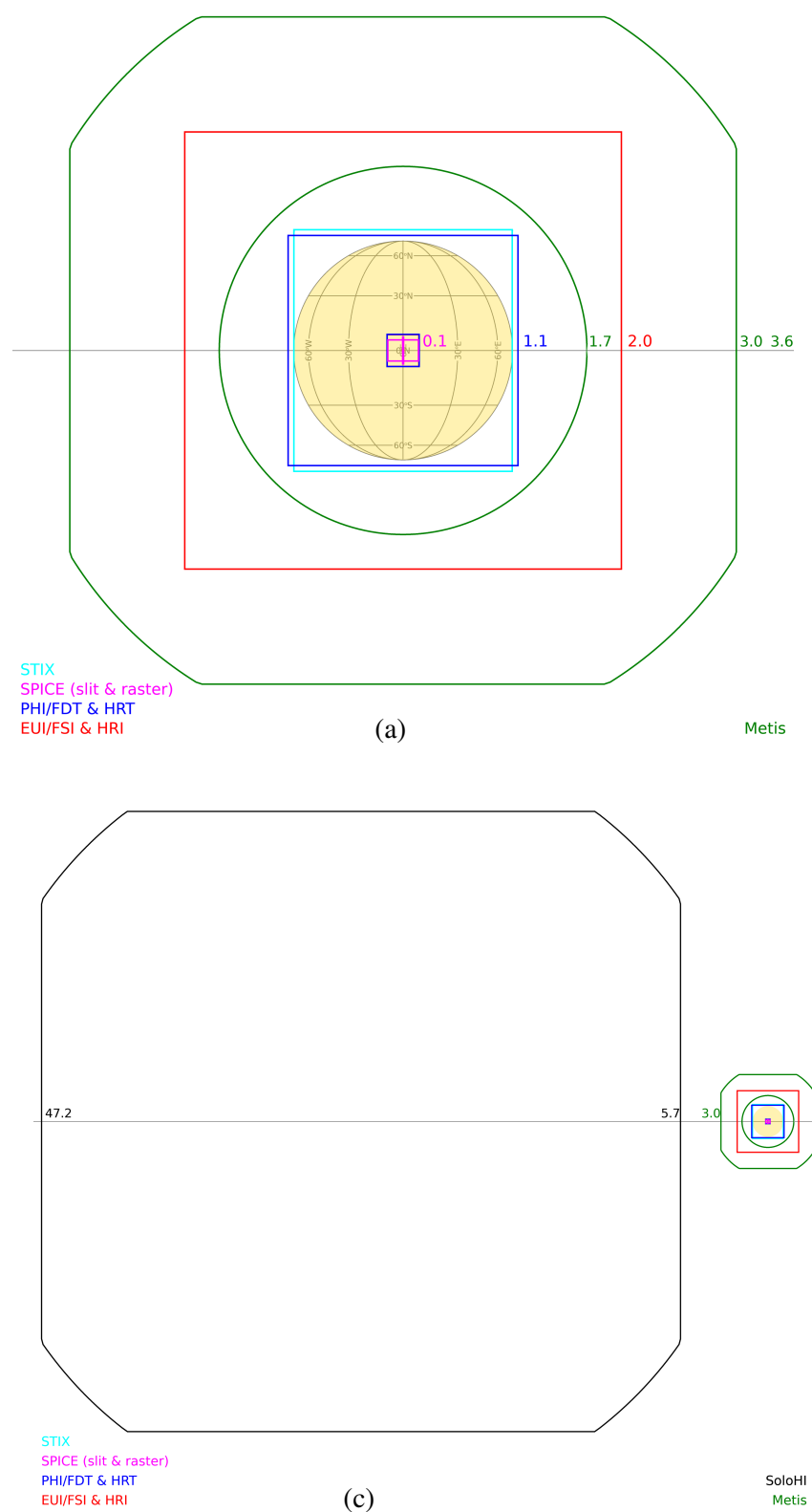

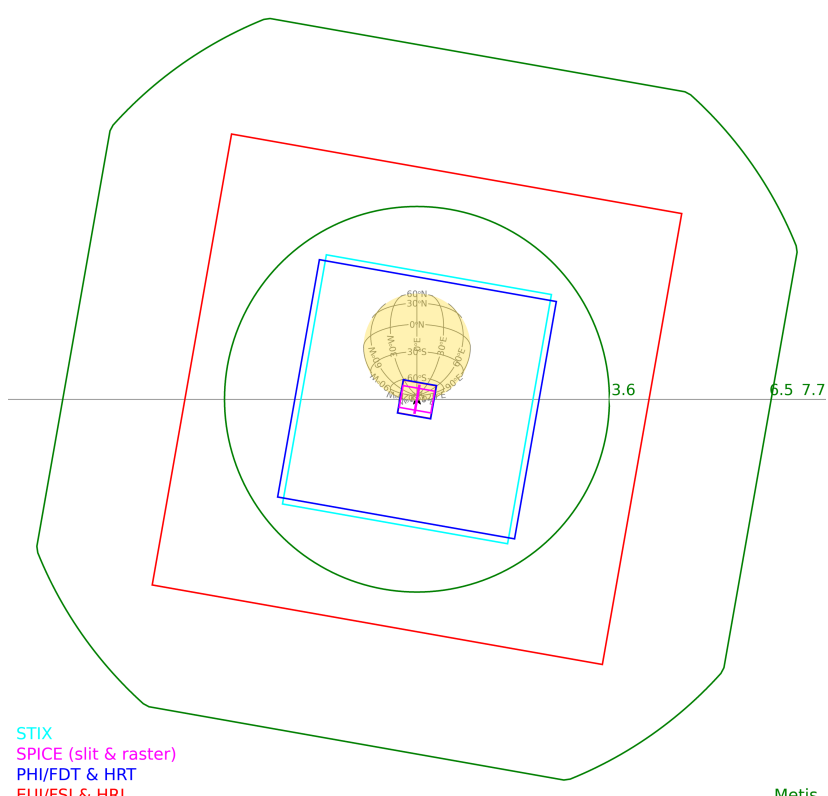

(b)

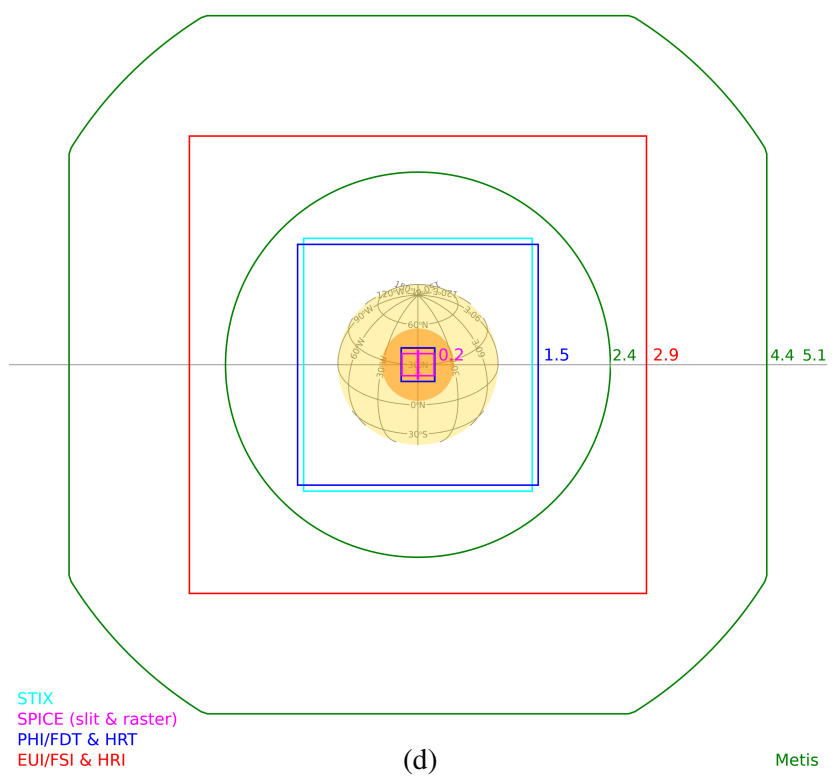

Fig. 1. Fields of view (FOVs) of the remote-sensing instruments in various orbital configurations and distances from the Sun. The extents of the FOVs are labelled in solar radii. The PHI/HRT, EUI/HRI ${ }_{174}$, and EUI/HRI $\mathrm{Ly}_{\alpha \alpha}$ FOVs are completely overlapping. In the cases where off-points are limited by the Metis safety constraint, the area on the solar disc within which off-pointing is allowed is highlighted in orange (see e.g. $(d))$. $(a)$ Closest perihelion at $0.28 \mathrm{AU}$, disc centre pointing. (b) Off-point to solar pole, at $0.6 \mathrm{AU}, \mathrm{S} / \mathrm{C}$ rolled $10^{\circ}$ with respect to solar north. (c) All RS FOVs, incl. SoloHI, at $0.3 \mathrm{AU}$ perihelion. (d) Maximum latitude case $\left(30^{\circ} \mathrm{N}\right)$, at $0.4 \mathrm{AU}$.

several (if not all) instruments adjusting their observation modes and parameters to serve one common science objective. Solar Orbiter's mission level and long-term planning will be performed at a SOOP level rather than on the level of individual instrument commands. Once the common campaigns have been scheduled and the involvement of each instrument defined, the shorter-term planning will start consolidating the command timeline for each instrument based on the agreed plan. The SOOP concept will be especially relevant for the RS payload as most instruments have many different observation modes which typically vary significantly in data output depending on the science goal being addressed.

Mission planning is driven by the six-month planning periods after each of the European Space TRACKing (ESTRACK) network User Group meetings. There are several levels of Solar Orbiter planning cycles (Sanchez et al., in prep.), as summarised here:

1. Mission Level Planning (MLP): the Solar Orbiter Science Working Team (SWT) defines top-level science activities for the entire mission (Science Activity Plan, SAP), as well as detailed science goals for each orbit.

2. Long Term Planning (LTP): given input from the SWT, the SOWG defines a coherent mission-level observing plan for a given orbit after ground station allocation has been fixed; it is assisted by the Science Operations Centre (SOC) at the European Space Astronomy Centre (ESAC), Madrid, which provides detailed information on the resources available. Science activities defined in the SAP are translated into SOOPs for a six-month 
period ( $\sim 1$ orbit), planned at least six months before the start of the period. A SOOP is a collection of instrument operations that belong together, i.e., that serve a common science goal.

3. Medium Term Planning (MTP): the instrument teams translate the SOOPs for a six-month period into Instrument Operations Requests (IORs), which provide details about all telecommands (TC) that will be sent to the S/C. The MTP is completed at least 4 weeks before execution, and fixes $\mathrm{S} / \mathrm{C}$ resources, instrument modes, and default pointing.

4. Short Term Planning (STP): this cycle covers 1 week and is planned approximately 1 week before execution. This is the last opportunity to modify instrument operation modes. It generates detailed schedules of commands for S/C and payload.

5. Very Short Term Planning (VSTP): this will be scheduled for a subset of RSWs only (agreed in the LTP) and allows updates to the S/C fine pointing (pVSTP) and/or limited updates of the instrument observations (iVSTP). Update requests are possible once every 24 hours, and the time between the request and the execution is no less than 3 days.

\section{2. "Hunting" for the "big event"}

Two of the four overarching science questions for Solar Orbiter (Müller et al. 2013) explicitly depend on observations of solar eruptions; namely:

- How do solar transients drive heliospheric variability?

- How do solar eruptions produce energetic particle radiation that fills the heliosphere?

The RS instruments will address these questions by returning observations of the source regions and early phases of the eruptions, whose interplanetary effects will be sampled by the IS instruments, at least for some eruptions. However, obtaining comprehensive observations of major eruptions with the RS payload is challenging for two reasons:

- Temporally: the RSWs comprise only one-sixth of the total orbit, meaning that about five-sixths of the eruptions will be missed. In addition, there is insufficient TM allocation for highresolution instruments like EUI and PHI to operate throughout the RSWs with a sufficient imaging cadence.

- Spatially: the FOVs of EUI/HRI $174, \mathrm{EUI} / \mathrm{HRI}_{\mathrm{Ly} \alpha}$, PHI/HRT, and SPICE cover an area 7 (at $0.6 \mathrm{AU}$ ) to 31 (at $0.28 \mathrm{AU}$ ) times smaller than the full solar disc, indicating a high likelihood that, with pre-defined pointing, many eruptions will be missed. This is not a concern for the wide FOV telescopes (STIX, Metis, SoloHI, EUI/FSI and PHI/FDT).

Clearly, catching eruptions "by chance" with the highresolution telescopes of Solar Orbiter is very unlikely, even over the full mission lifetime. A study of the Hinode space mission flare catalogue carried out by Watanabe et al. (2012) found that the two narrow FOV instruments on the mission observed $<25 \%$ of the flares. This is a consequence of having to choose the eruption target (there may be many possible pointing options during solar maximum). The Hinode space mission is in Earth orbit and hence the pointing can be adjusted or changed within a few days. There will be less flexibility in the Solar Orbiter case. However, the experience of the Hinode mission suggests that there is a higher chance of successfully choosing the target during solar minimum, since there is likely to be only a single active region on the disc at any one time. Solar Orbiter will be starting its science phase in the solar minimum period. The experience gained early on will help with target selection later on during the rising phase of the cycle.

Taking into consideration these challenges, we have developed specific strategies to increase the chance of making multi-instrument eruption observations. In appropriate observation campaigns, the $\mathrm{S} / \mathrm{C}$ pointing can be updated up to 3 days before the observations. This is similar in timescale to the Hinode case which usually finalises pointings two days in advance. Analysis of prior synoptic data from other sources is key to the success of capturing eruptions. An example which has been extensively used by several missions is the Max Millenium program that issues Major Flare Watches based on solar data across the electromagnetic spectrum to assess the probability of a large solar flare. Missions like Hinode replan observations based on a Major Flare Watch, which has been effective. Bloomfield et al. (2016) describes the success rate of this process. Several instruments have data trigger mechanisms that autonomously identify which data sets contain eruptions (Sect. 3), as was successfully demonstrated for the Sun-Earth Connections Coronal and Heliospheric Investigation (SECCHI, Howard et al. 2008) suite of instruments on the Solar-TErrestrial RElations Observatory (STEREO, Kaiser et al. 2008) mission. Some instruments can overwrite data which did not emanate from trigger events. All instruments can receive and communicate to such identifications onboard.

The means of communication between instruments is described in Sect. 3. Three instruments have triggers in their onboard systems. The EUI has a flare trigger responding to intensity enhancements in EUI/FSI; STIX has a flare trigger responding to flares in the X-ray; and Metis has a CME trigger that responds to enhancements in white light emission off-limb. All instruments can share their coordinates and timings of the events and respond appropriately. One example of a response could be to prioritise these data for download. It is important to remember that the three triggers respond to different parts of the solar atmosphere and hence the response times will be different - and indeed all three instruments may not respond to the same event.

\subsection{Pointing strategy}

The whole RS payload is co-pointed and the four high-resolution telescopes (EUI/HRI 174 , EUI/HRI $\mathrm{Ly}_{\alpha}, \mathrm{PHI} / \mathrm{HRT}$, SPICE) cover only part of the solar disc even when far from the Sun (Fig. 1d). Fulfilment of several of the Solar Orbiter scientific objectives thus requires that the $\mathrm{S} / \mathrm{C}$ be off-pointed to the relevant targets. For example, the $\mathrm{S} / \mathrm{C}$ will have to track active regions considered likely to produce major events to maximise the chances of observing large eruptions with the high-resolution telescopes (see Sect. 2.2). Pointing at the poles is required for SPICE Doppler measurements of the fast wind outflows from the polar coronal holes (SPICE Consortium 2020), or PHI measurements of the polar magnetic field strength and seismic diagnostics of the subsurface flows (Solanki et al. 2020). Coordinated observations with the IS payload will require the RS payload to be pointed at the sources of the solar wind, which may not be located near disc centre. For this purpose, the operations team will use state-of-the-art modelling (Rouillard et al. 2020) to predict the location of the solar wind source regions. One possible method to track solar plasma from its source to the solar wind is to compare abundance measurement made remotely by SPICE at the surface and in-situ by the Solar Wind Analyzer (SWA, Owen et al. 2020). Since this does not require high cadence, a possible approach to maximise the chances of observing the correct regions will be to assemble synoptic (e.g. daily) meridian mosaics, thus eliminating the latitudinal uncertainty.

The necessity to off-point needs to be balanced against the safety of the Metis coronagraph. To prevent direct sunlight from reaching the focal plane, the Metis door will have to be closed 
whenever the $\mathrm{S} / \mathrm{C}$ points further than $0.1 R_{\odot}$ at $0.28 \mathrm{AU}$ linearly up to $1 R_{\odot}$ at $0.51 \mathrm{AU}$, which is the $\mathrm{S} / \mathrm{C}$ off-pointing capability limit. For example, the orange disc in Fig. 1d marks the Metis safety limit at 0.4 AU. Off-points larger than its safety limit will result in Metis being closed, thus preventing joint observations with the other instruments. This may be mitigated by Metis observations right before and after the off-point manoeuvres.

\section{Inter-instrument communication (Service 20)}

An inter-instrument communication (IIC) service has been established on Solar Orbiter to allow instruments to cross-share a limited set of parameters, summarised in Table 2. The IIC is implemented through the use of a dedicated TM packet that each instrument sends to the computer (OBC) with a fixed cadence. All received parameter data are gathered in one data pool. These are extended with extra parameters based on S/C events (like pointing information) and are then communicated back from the OBC to the instruments through the so-called Service $20 \mathrm{TC}$ packets. This service will mainly be used to pass on event detection flags which may sequentially trigger actions in the receiving instruments to safeguard or prioritise data which are likely to include a solar event. In addition, Service 20 packets will include an instrument-level heartbeat that can be used to identify stale data. The service is available to all payloads, but due to the different nature of IS compared to RS instruments and the time delays between activity observed on the Sun and its characteristics measured in-situ, each group of instruments will typically only react to triggers from its own group.

The RS instruments have agreed to generate IIC TM packets at a cadence of $1 \mathrm{~Hz}$. The only exceptions are SPICE and SoloHI which currently do not plan to participate in this inter-instrument communications mechanism, but will keep the capability to use Service 20 in the future. The STIX, Metis, and EUI plan to broadcast event flag information, which is detailed in the paragraphs below. The PHI will share S/C jitter information based on its automatic Image Stabilisation System (ISS, Solanki et al. 2020). The S/C-provided parameters will also include information on pointing stability (e.g. on the occurrence of non-planned thruster firings) and will allow instruments to assign low priorities to images taken during these events.

\subsection{The detection algorithm of the Extreme Ultraviolet Imager and actions based on inter-instrument communication content}

The EUI will run detection algorithms on the images generated by FSI in both wavelengths. It will broadcast, both for EUI/FSI 304 and EUI/FSI 174 , whether an event has been detected, the time and location (with respect to the instrument boresight) of the detection, and a rough estimate of the brightness of the event. The detection algorithm is based on the SoFast activity detector $^{1}$ (Bonte et al. 2013). It uses thresholds on macropixels and a set of rules to minimise the number of false detections. It must be noted however that due to the limited space in the memory of EUI and its automated onboard data management, an EUI detection does not necessarily mean the EUI images make it to the ground. If later a more significant event is detected, it may potentially overwrite the earlier ones. Also, the detection parameters will need to be adjusted in-flight to take into account the properties of the real data, and any effects, for example those caused by the varying size of the Sun.

\footnotetext{
1 http://sidc.be/sofast
}

In turn, EUI will listen to flags broadcast by STIX and Metis. It will combine the information from these two instruments with its own event detection using simple logic (e.g. STIX sees a flare and EUI sees a flare in the same region) to prioritise its data. Also, EUI is planning on using the PHI jitter information for data prioritisation.

\subsection{The CME-detection algorithm of Metis and actions based on inter-instrument communication content}

The Metis CME-detection algorithm monitors the total intensity in eight sectors nominally aligned to the instrument frame of reference. Metis will run its event detection on difference images of the same polariser position. The algorithm is based on that implemented on SECCHI, COR2 (Howard et al. 2008). The COR2 algorithm uses macro-pixels and total brightness images computed onboard from double exposures. As for the other instruments, the detection parameters will need to be finetuned in flight and the effect of the varying angular size of the Sun (size of the FOV with respect to the solar disc size) on the detection needs to be assessed. This is particularly relevant for Metis as the range in heliocentric distance covered by the Metis FOV varies with the S/C-Sun distance. For example, Metis will raise its CME-event flag higher in the corona when the $\mathrm{S} / \mathrm{C}$ is at $0.5 \mathrm{AU}$ than when it is at $0.28 \mathrm{AU}$. In addition, the stray-light pattern may also vary.

In addition to its own detection algorithm, Metis can also react to the EUI and STIX flags. In particular, Metis will listen to off-limb events detected by EUI/FSI 304 and on-disc flares detected by STIX. The reaction thresholds to those flags are programmable and will be fine-tuned in flight. Moreover, a time delay can be set for the Metis reaction to those on-disc or neardisc events to account for the CME transit time through the inner region of the solar corona up to the inner edge of the Metis FOV. Once a CME flag is activated, either internal or external, the detection of an event will make Metis switch to its special CME observing mode.

\subsection{The detection algorithm of the Polarimetric and Helioseismic Imager and actions based on inter-instrument communication content}

The PHI will provide four parameters containing the S/C pointing stability obtained from the correlation functions of the PHI ISS (Solanki et al. 2020). During dedicated RSWs, PHI will listen to the STIX flare trigger for possible white light flares. If triggered, PHI will autonomously switch to a special science operation mode tailored to obtain high-cadence continuum images in the $617.3 \mathrm{~nm}$ spectral range interlaced with magnetograms. In addition, PHI will read from the data pool of information provided by $\mathrm{S} / \mathrm{C}$ on its stability, PHI's heat rejecting entrance windows, and the instrument doors. Science images that are acquired during times where Service 20 data indicates nonnominal pointing accuracy will be marked.

\subsection{The detection algorithm of STIX and actions based on inter-instrument communication content}

The STIX will use a dedicated sub-collimator (grid) to detect flares and broadcast the corresponding information to indicate the current level and location of solar activity with 1 arcmin location accuracy. The flag will be updated no more frequently than once every 4 or $8 \mathrm{~s}$ (configurable by TC). The latency will also 
Table 2. Summary of inter-instrument communications between the RS instruments.

\begin{tabular}{lll}
\hline \hline Instrument & Broadcasts & Reacts to \\
\hline EUI & FSI events timing and location & STIX and Metis flags and PHI jitter data \\
Metis & CME timing and location & EUI and STIX flags \\
PHI & S/C pointing stability & STIX flags \\
SoloHI & - & - \\
SPICE & - & - \\
STIX & X-ray flares timing and location & - \\
\hline
\end{tabular}

Notes. See Sect. 3 for details.

Table 3. Synoptic observations planned outside the nominal Remote Sensing Windows.

\begin{tabular}{llc}
\hline \hline Instrument & Observations/data products & Data volume \\
\hline EUI & 15 min cadence EUI/FSI 174 & 4.2 \\
Metis & 30 min cadence visible light & 6.9 \\
PHI & $\begin{array}{l}\text { Daily Full Sun } B_{\text {LOS }} \text { and con- } \\
\text { tinuum images }\end{array}$ & 3.3 \\
SoloHI & $\begin{array}{l}\text { 30 min cadence } 2.5^{\circ} \text { wide equa- } \\
\text { torial and latitudinal swaths }\end{array}$ & 3.2 \\
SPICE & $\begin{array}{l}\text { Daily First Ionisation Potential } \\
\text { bias map }\end{array}$ & 3.4 \\
STIX & Nominal operations (Table 1) & 33 \\
\hline
\end{tabular}

Notes. The data volume is given as a percentage of each instrument's TM allocation.

be 4 or $8 \mathrm{~s}$, as configured. The STIX will not read or react to messages from other instruments; there is no need to do so because of its mono-mode observing and post-facto data selection.

\section{Out-of-RSW observations}

The nominal strategy of RS windows centred on the perihelia and maximum latitudes maximises the scientific return of the mission during these unique moments, but also implies that the connection between the RS and the IS observations is effective only during one-sixth of the mission duration. In order to increase the duration of the joint observations, the RSWG investigated the possibility for the RS instruments to dedicate a fraction of their TM allocation to perform synoptic "out-of-RSW" observations that will provide the contextual information necessary to enable connection science throughout orbits, while maintaining a low-resource/low-impact profile and avoid violating the Electro-Magnetic Compatibility (EMC) requirements of the IS instruments.

It was found that, while added late into the development of the mission ground segment, out-of-RSW observations could be made almost resource neutral for the ground operations teams as long as they fit into the low-latency data volume (Sect. 6). Each RS instrument therefore designed a "synoptic" type program that can be run continuously to provide basic contextual information (Table 3) without impacting their core objectives.

\section{Inter-calibration}

Achieving the Solar Orbiter science objectives relies on the close coordination between the RS instruments. This requires that the data from the various RS instruments be directly comparable in terms of temporal and spatial localisation. The former is ensured via the SOOP mechanism (Sect. 2.1). The latter implies accurate co-alignment between the various telescopes and is discussed in Sect. 5.2. Moreover, the quantitative analysis requires a reliable inter-instrument relative radiometric calibration during flight as the various instruments will age and deviate differently from the ground calibration (Sect. 5.3).

\subsection{Remote sensing checkout windows}

Unlike previous RS platforms for the observation of the Sun, the first science observations will take place at the end of a Cruise Phase (CP) lasting 1.8 years. Furthermore, the commissioning activities performed during the first months of the mission will not test the instruments in an environment representative of the thermal and radiation conditions of the perihelia. It will therefore be necessary to monitor the instrument health and behaviour during the CP to ensure their optimal performance at the start of the Nominal Mission Phase (NMP). For this purpose, four remote sensing checkout windows (RSCWs) have been planned through the CP. Each of these windows lasts a few days during which each instrument will characterise its response to the changing environment. In addition, coordinated observation campaigns will take place to monitor the relative pointing of the instruments (Sect. 5.2).

\subsection{Co-alignment among RS instruments}

Telescope co-alignment requires the determination of three angles (pitch, yaw, and roll) for each telescope defining the deviation from a common reference frame. The choice of reference frame is not important for the following discussion (it could be the $\mathrm{S} / \mathrm{C}$ reference frame), but for clarity we can assume that it is centred on the $\mathrm{S} / \mathrm{C}$, with the roll axis pointing towards Sun center. In addition, co-registration requires the knowledge of the plate scale for each detector, and eventually the knowledge of the optical distortion across the FOV. The instruments have been co-aligned on the ground during $\mathrm{S} / \mathrm{C}$ integration so that the lines of sight of the high-resolution channels will be in flight within the specified two-arcminute cone (Fig. 2). The values take into account the measurements of the instrument lines of sight with respect to their reference cubes, the relative orientations of all the reference cubes, the measurement errors, and the modelled thermo-elastic distortions. While this ensures sufficient overlap of the FOVs, the lines of sight are expected to move within that cone due to the changing thermal environment, thus requiring regular monitoring of the relative orientations of the telescopes. In-flight verification of the relative pointing of the instruments will be performed several times during CP. Although they will not cover the full range of temperatures encountered during the NMP, these tests will allow correlation with the thermal models of the instruments. 


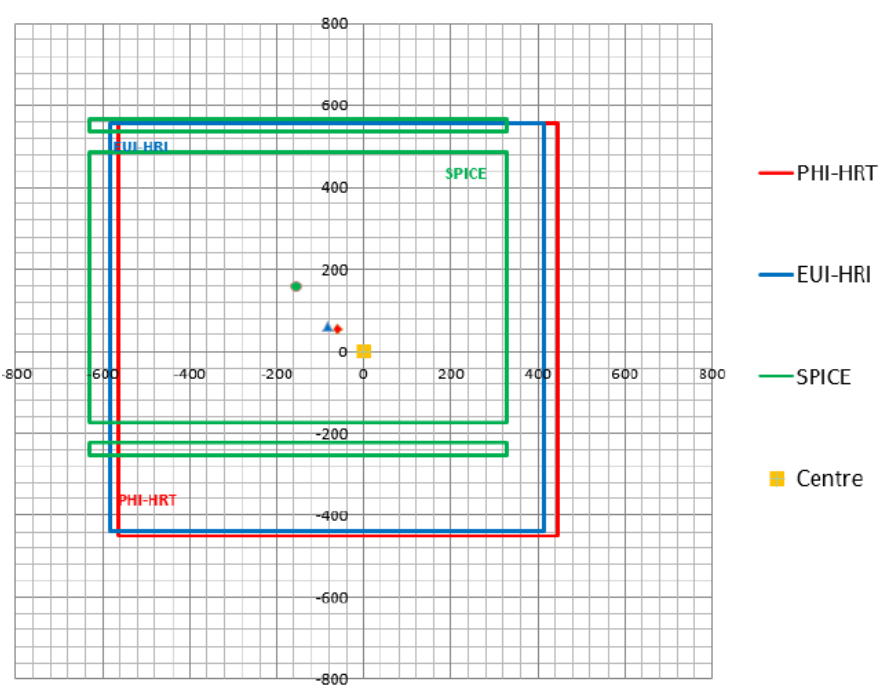

Fig. 2. Estimated worst-case co-registration configuration of the highresolution fields of view based on the pre-flight measurements (in arcsec). All known sources of misalignment, both internal and external to the instruments, are included. The two horizontal bars in the SPICE FOV are the co-registration dumbbells at the ends of the slit (SPICE Consortium 2020).

\subsubsection{Using stars and planets}

Stars and planets provide a reliable reference with which to determine all the co-alignment parameters. SoloHI and the VL channel of Metis will observe stars in all their images and their orientation can be determined accurately with respect to the reference frame from the $\mathrm{S} / \mathrm{C}$ orbital position. This is also true of the Metis ultraviolet (UV) channel that will be able to detect stars with significant emission around $121.6 \mathrm{~nm}$, when such stars are present in the FOV. These bright UV stars will also be visible in the VL channel. Tracking of stars across the FOVs can also be used to derive the plate scale and optical distortion (Thernisien et al. 2006). Similarly, two transits of Mercury in front of the corona (in January 2023 and May 2027 at about 1.7 and $1.1 R_{\odot}$, respectively) will be observable by FSI. These events may become grazing or disc transits depending on the exact launch date and trajectory, in which case they would also be observable by PHI/FDT and the highresolution telescopes (SPICE, EUI/HRI $\mathrm{H}_{174}, \mathrm{EUI} / \mathrm{HRI}_{\mathrm{Ly} \alpha}$ ). They will provide unique opportunities to determine the pointing, roll (Couvidat et al.2016), plate scale (Auchère \& Artzner 2004), and distortion for these instruments. Aside from these rare opportunities, these telescopes will have to rely on other means to monitor their pointing.

\subsubsection{Solar limb fitting}

Since the solar disc will always be visible in the PHI/FDT and FSI FOVs, the position of Sun center can be determined to sub-pixel accuracy by fitting the limb (e.g. Auchère et al. 1998, 2000). For FSI, a better precision will be achieved in EUI/FSI 174 because the limb is sharper at $17.4 \mathrm{~nm}$ than at $30.4 \mathrm{~nm}$ (Auchère et al. 1998; Defise et al. 1999). EUI/FSI 304 is co-aligned by design with EUI/FSI 174 as the switch between one and the other occurs by swapping filters without optical power. Offpoint manoeuvres can be used to determine the plate scale of these instruments (Auchère et al. 2000) provided that the $\mathrm{S} / \mathrm{C}$ attitude is known to a sufficient accuracy. For PHI, the plate scale can also be obtained relative to the photospheric diameter.
This does not apply to FSI because the altitude of the limb at extreme ultraviolet (EUV) wavelengths is not accurately known. Roll manoeuvres can be used to determine the aspect ratio of the pixels and thus the isotropy of the plate scale.

The STIX is a full Sun instrument but limb fitting cannot be used with X-ray images. The instrument is however equipped with an internal optical system able to determine the instrument pointing with respect to Sun centre (by a type of analogue limb fitting) to a precision of 4 arcsec (Krucker et al. 2020).

While constant monitoring of the direction of Sun centre is possible by limb fitting, the method is insensitive to the roll angle, which therefore has to be determined from observations of stars or planets (Sect. 5.2.1). Finally, the pointing of EUI/HRI $\mathrm{I}_{174}$, EUI/HRI $\mathrm{Ly}_{\mathrm{L} \alpha}$, and SPICE may be determined from limb observations made during $\mathrm{S} / \mathrm{C}$ off-points and comparison with simultaneous FSI images. A similar comparison between PHI/HRT and PHI/FDT will be made difficult by the time required for thermal stabilisation of PHI/HRT following a PHI/FDT observation.

\subsubsection{Cross-correlation}

Cross-correlation can provide the relative plate scale and the three angles necessary to co-register the data from two instruments. Therefore, for the instruments that do not routinely see stars or the solar limb, the pointing with respect to the common reference frame can be obtained by cross-correlation with one that does, provided that their passbands are similar or correlate well with each other. Due to their very similar passbands, it is possible to cross-correlate nearly simultaneous EUI/HRI 174 and EUI/FSI ${ }_{174}$ images to a high accuracy (typically 0.1 FSI pixel, i.e. $0.45 \operatorname{arcsec}$ ). Likewise, the EUI/HRI $\mathrm{Ly}_{\alpha}$ images can be coaligned to a similar accuracy with the EUI/FSI 304 as the two bands are highly correlated (Gordino et al. 2020).

The pointing of PHI/HRT with respect to PHI/FDT was measured on the ground (see Solanki et al. 2020, this volume). However, the switch between the two telescopes requires a thermal settling time that is estimated to be of the order of hours (to be confirmed during flight). Thus, the repeatability of relative pointing of the two telescopes will be known only after the first two checkout windows. PHI/HRT images cannot be crosscorrelated against PHI/FDT ones since the two channels do not image simultaneously.

The images taken by PHI/HRT may however be correlated against SPICE raster images. The C I continuum around 102$104 \mathrm{~nm}$ is formed low enough in the atmosphere (above the temperature minimum, at a height of around $1000 \mathrm{~km}$ according to Vernazza et al. 1981) that images in this spectral emission should cross-correlate quite well with maps of the absolute longitudinal field (see Fig. 3).

The pointing of SPICE itself can be determined by crosscorrelating Lyman $\beta$ rasters with EUI/HRI $\mathrm{Ly}_{\alpha}$ images (see, e.g. Fig. 2 of Tian et al. 2009), or $102.8 \mathrm{~nm} \mathrm{Fe} \mathrm{X} \mathrm{and} \mathrm{77.0} \mathrm{nm} \mathrm{Ne} \mathrm{VIII}$ rasters with images from EUI/HRI 174 . Since both spectral channels are fed by the same slit, co-aligning the two channels consists of identifying the end points of the slit on the detector for each channel.

Table 4 summarises the methods used to co-align the various instruments.

\subsection{Photometric inter-instrument calibration}

It is predicted that relative cross calibration of the RS instruments will be difficult because of the quasi absence of common lines or passbands between the instruments. Observation of 

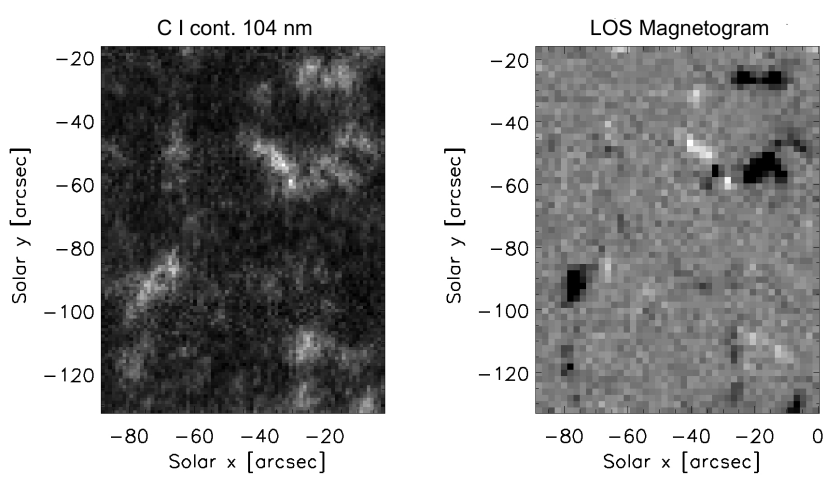

Fig. 3. Left: rastered (east to west) image of the quiet Sun obtained with the SUMER spectrograph aboard SOHO in the $\mathrm{C}$ I continuum around $104 \mathrm{~nm}$. Right: line of sight magnetogram of the same area taken by the MDI instrument aboard SOHO. Data are clipped to the -85 to $+85 \mathrm{G}$ range. The magnetogram was acquired around the time SUMER completed the 13 minutes scan, on 17 April 2009 around 09:38 UTC.

bright FUV stars (e.g. hot O and B stars) can be effectively used to cross-calibrate EUI and Metis at Lyman $\alpha$. The same stars appear to be bright enough to also be detected by the SPICELW channel. Since the EUI or EUI/HRI $\mathrm{Ly}_{\alpha}$ observations of stars can be made only during offpoints, the Metis door will have to be closed when these observations are made below $0.55 \mathrm{AU}$. In these cases, the stars will be observed by Metis before and/or after off-points, provided enough time for repointing to disc centre before the end of the transit through the Metis FOV. It is possible for EUI/HRI 174 and EUI/FSI 174 to be cross-calibrated with SPICE using the $102.8 \mathrm{~nm}$ line of Fe X. Cross calibration of EUI/FSI 304 and EUI/HRI $\mathrm{Ly}_{\alpha}$ may be more problematic, as SPICE only observes Lyman $\beta$.

The most effective way to monitor the radiometric response of instruments in space is by comparing them to stable and wellknown calibration sources such as calibration rocket flights and onboard calibration lamps. As the former are not foreseen and the latter are not included in any of the instruments, the only possibility is to use natural sources.

For visible-light instruments, the task is performed easily either against the solar quiet photosphere (PHI) or against reference stars (Metis VL and SoloHI). Monitoring the radiometric response of instruments becomes more difficult for shorter wavelengths. Instruments detecting radiation above the hydrogen absorption edge can be calibrated against bright $\mathrm{B}$ and $\mathrm{A}$ main sequence stars as they provide stable and intense flux at wavelengths above $100 \mathrm{~nm}$ (Mihalas \& Binney 1981). A discussion on several UV stars suitable for calibration can be found in Snow et al. (2013). Figure 4 shows the spectrum of $\alpha$ Virginis, which has substantial emission down to $100 \mathrm{~nm}$. Figure 5 shows that bright UV stars should be easily detectable with the SPICE spectrograph. A similar result is expected for the EUI/HRI Lya telescope while equivalent calculations show the same also for the Metis UV channel. $\alpha$ Leo and $\sigma$ Sgr are particularly useful as they are occulted a few times by the solar disc during the cruise phase, and the nominal and extended mission phases.

A further check is provided by observing the H I Lyman $\alpha$ (EUI/HRI $\mathrm{Ly}_{\mathrm{L} \alpha}$ ) over Lyman $\beta$ (SPICE) ratio in the quiet Sun. This ratio is remarkably constant, particularly in the regions with lower magnetic flux (Tian et al. 2009). The situation is more complex for the EUV instrumentation, namely EUI/FSI and EUI/HRI ${ }_{174}$, as any stellar signal in the EUV is too faint to be detectable. The radiometric throughput of these channels can only be checked relative to the average values in selected regions (e.g. the quiet Sun) with high uncertainties or against other instruments that can be more closely monitored. For instance, the EUI EUI/FSI 304 could be checked against the Metis Lyman $\alpha$ channel, since their FOVs overlap significantly (Fig. 1).

\subsection{Calibration stability}

Keeping throughout the mission the calibration that has been achieved during ground calibration campaigns is a major undertaking that is intimately related to the cleanliness of the $\mathrm{S} / \mathrm{C}$. The RS instruments with their optical components are exposed to solar UV irradiation, which contributes to degradation due to polymerisation when organic material is deposited on them. This degradation is strongly wavelength dependent. Particular emphasis was therefore put on the molecular cleanliness of the payload and S/C during design and build activities. The cleanliness requirements of the payload instruments were established very early in the program and manifested as interface requirements to be fulfilled by instruments and S/C. All activities needed to minimise particulate and molecular contamination were carried throughout Assembly, Verification, and Validation (AIV) processes (García-Marirrodriga et al. 2020). For example, all flight hardware went through a bake-out process and the fully assembled $\mathrm{S} / \mathrm{C}$ was finally subjected to a vacuum-thermal cycling and out-gassing process with monitoring and verification of the cleanliness with unprecedented accuracy. In addition, the S/C carries a purge gas distribution system to supply all payloads with clean gas during all ground activities, including transport, up to launch. Some activities to minimise molecular contamination have been implemented by design and they have impact on the operations of the payload:

- Heat Shield doors. All RS instruments but SoloHI have their apertures passing through openings in the Heat Shield. With the exception of STIX, each opening has its own door mechanism activating a shield that can open and close the apertures individually. Such a shield will reduce UV exposure of the instrument's first optical surfaces during non-operational phases of the flight. During the commissioning phase, Heat Shield doors can be opened at the request of each instrument. During coordinated observations, the doors must be opened well in advance to achieve thermal equilibrium.

- Instrument doors (SPICE, EUI). In addition to Heat Shield doors, the UV instruments SPICE and EUI have their own aperture door mechanisms that allow them to open and close the aperture at any time. These doors shall stay closed during any period of long non-operational phases. The doors are designed with a motor drive mechanism and a sliding door, closing the instrument aperture with a minimal gap such that no UV light can enter. The three EUI telescopes each have a door mechanism, and the door of the EUI/FSI carries a circular shield that can be placed in the aperture in such a way as to occult the solar disc, enabling FSI to be used as a quasi-coronagraphic imager. The doors keep the instruments closed during ground activities. They allow the maintainance of a small overpressure provided by continuous purging with clean gas. The doors remain closed during the first phases of the mission to prevent any ingress of contamination. They will be opened when it has been verified by the Contamination Monitoring System (CMS) that molecular deposition at the apertures is low enough and the risk of molecular contamination is minimal.

- Contamination Monitoring System. The Contamination Monitoring System consists of two thermally controlled quartz crystal micro-balances (QCM) that are powered and operated by the SPICE instrument. One is located next to the SPICE 
Table 4. Inter-instrument spatial co-alignment matrix.

\begin{tabular}{lllll}
\hline \hline & $\begin{array}{l}\text { Common reference } \\
\text { frame }\end{array}$ & EUI EUI/HRI 174 & EUI EUI/HRI $_{\text {Ly } \alpha}$ & PHI/HRT \\
\hline PHI/FDT & $\begin{array}{l}\text { Limb fitting } \\
\text { Planet transit }\end{array}$ & & & \\
\hline Metis & VL stars & & & \\
& Planet transit & & & \\
& UV stars & & & \\
\hline SoloHI & Stars, planets & & Cross corr of 304 & \\
\hline EUI FSI & Limb fit (174) & Cross corr. of & & \\
& & 174 images & Corr. C I scans \\
& Planet transit & H I, Fe X, Ne VIII & Cross corr. with & with $B_{\text {LOS maps }}$ \\
\hline SPICE & & scans vs. 304 and & Lyman $\beta$ scans & \\
& & & &
\end{tabular}

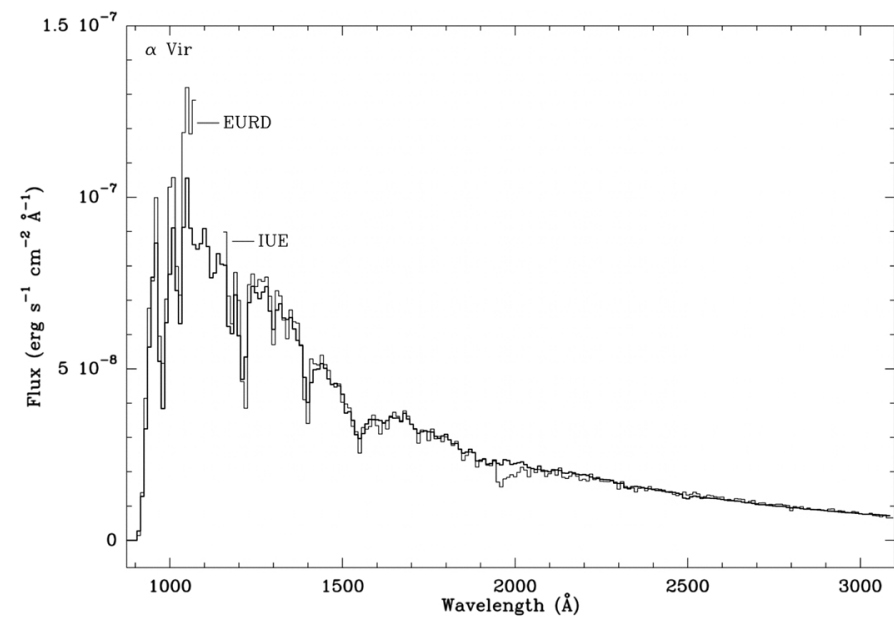

Fig. 4. Spectra of $\alpha$ Vir as observed by EURD $(\lambda<107 \mathrm{~nm})$ and IUE $(\lambda>116 \mathrm{~nm})$, superposed on the Kurucz model (heavy line) scaled to IUE data. Figure taken from Morales et al. (2000), reproduced with permission.

aperture door (CMS1) while the other is mounted next to the SWA-HIS instrument (CMS2) behind the Heat Shield. The two QCMs measure the deposition of condensable material from the environment. They serve as a diagnostic tool to assist in the planning of opening the protective doors and to characterise the out-gassing of the $\mathrm{S} / \mathrm{C}$ during the mission.

- Protective devices: Metis protection cap, PHI entrance filter, SPICE mirror and ion deflector, and SoloHI door. In order to minimise the particle contamination during ground and launch activities, an ejectable sealing cap is mounted at the Metis entrance aperture behind the Heat Shield door. The sealing cap ejection system is a one-shot mechanism that will release and eject the protection cap. It is planned to be operated at the very beginning of the mission. After the ejection, the Metis aperture remains open and the Heat Shield door is fundamental for the safety of the Metis instrument as it must be closed when the $\mathrm{S} / \mathrm{C}$ is pointing off Sun center, since the coronagraph is designed specifically to operate in Sun-centred position. The two apertures of the PHI instrument have no extra door mechanism because they are protected by the Heat Rejection Entrance Windows, which are spectral interference filters mounted inside

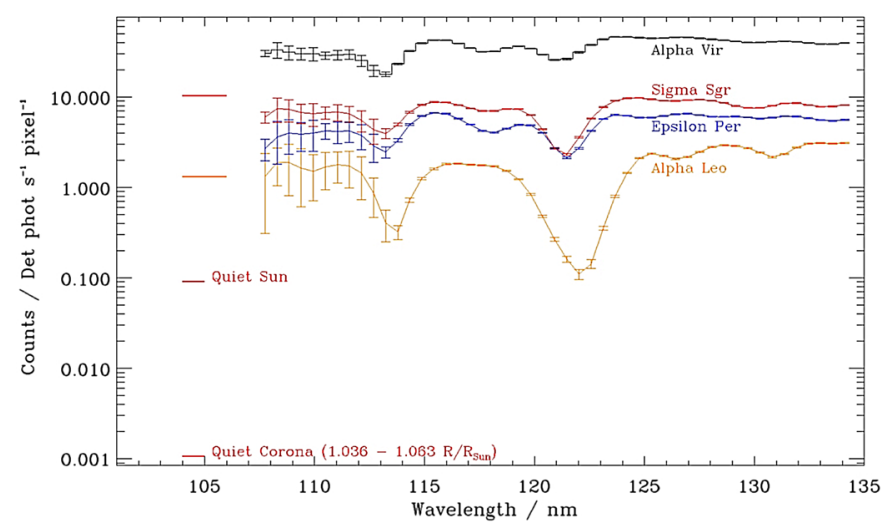

Fig. 5. Detected photons per second per pixel expected by the SPICE spectrograph for the displayed UV stars. The calculations assume that all the flux entering the telescope, after accounting for the SPICE effective area, ends up in one pixel. More realistically, these values should be divided by ten. The expected levels for the quiet Sun disc emission and for the emission expected in the quiet corona above the limb are also shown for comparison.

the feedthroughs between the Heat Shield and the instrument aperture. These windows prevent contaminants and UV radiation entering the optical system of PHI. The SPICE primary mirror carries only a very thin reflective coating of $10 \mathrm{~nm}$ in thickness to reflect the vacuum-ultraviolet range of SPICE while it transmits most of the infrared and visible spectral range heating the mirror. Whenever the SPICE aperture is open, the instrument's ion deflector must be operational, with a high voltage applied to the plates that will deflect charged solar wind particles from their path towards the primary mirror. The SoloHI door covers the baffle area of the instrument during ground operations. It protects the sensitive baffle edges from accidental damage and dust from accumulating on the baffles. The door opening mechanism is a one-time actuation, and is released after launch when the contamination level is sufficiently low.

\section{Low-latency data}

As explained in Sanchez et al. (in prep.), scientific data generated onboard are assigned different downlink priorities. The need to define a set of high-priority data became clear when 
simulations of the fill state of the onboard Solid State Mass Memory (SSMM) revealed that the maximum capacity of the SSMM will likely be reached several times during the mission, resulting in high data latencies.

In order to minimise the effect of data latency on the instrument operations planning and performance checks, a minimal set of so-called low-latency (LL) data has been defined for each instrument. The LL daily volume has been necessarily restricted to $1 \mathrm{MB}$ per instrument ( $2 \mathrm{MB}$ in exceptional cases), to guarantee that it can be downlinked at each $\mathrm{S} / \mathrm{C}$ contact, even at times of poor communications links (at the far side of the Sun, close to $2 \mathrm{AU}$ from Earth) and taking into account that $\mathrm{S} / \mathrm{C}$ and instrument housekeeping data (HK) will be brought down first. The LL dataset fulfils three use cases:

1. It allows basic checks of instrument performance and science data quality (i.e. avoids the up to six-month delay in assessing instrument performance).

2. It allows data selection for those instruments with onboard data buffers or can help (re-)prioritise data in instrument internal memories.

3. It allows the pointing profile to be readjusted and/or retargeted when tracking solar features.

A special use case of remote-sensing LL data is the precursor dataset that will be acquired before the start of some RSWs. As described in Sanchez et al. (in prep.), several mission science objectives that require RS observations make it necessary to perform precursor observations before the start RS windows to enable the SOC to choose an optimal fine-pointing profile for the $\mathrm{S} / \mathrm{C}$ that is commensurate with the pre-planned science activity (related to LL use case 3 defined above). As this requires a fast turn-around time between data acquisition and S/C pointing updates, the data will be downlinked as part of the daily TM dump.

Table 5 lists the sets of low-latency data that have been defined for the RS payload. The numbers between square brackets refer to the use cases defined above. The table shows that in general the low-latency concepts adopted by RS instruments are more complex and less continuous than the approach of the in-situ instruments (see Walsh et al. 2020). This is partly due to the noncontinuous way of operating, and the variety of science goals assigned to the RS windows. For most instruments, the LL data volume and/or content is likely to vary over the mission and contains data which are specifically designed for the LL data stream. Nevertheless, at all times, the LL data need to fit in the small TM allocation to guarantee prompt retrieval. It must be noted that as a consequence of the extreme compression factors and/or low time resolution, the RS LL data may be unsuitable for scientific analysis. However, they are of high operational value; for example, for the evaluation of the conditions on the Sun on the far-side of Earth, the choice of the best target for the high-resolution instruments, the assessment of instrument performance and data quality, and for providing optimal onboard data management.

\section{Common FITS headers content}

Solar Orbiter science data are organised into two categories:

- remote sensing images and spectra which are stored in the Flexible Image Transfer Standard (FITS; Pence et al. 2010) format widely used within the astronomical community;

- and in-situ and radio data which are stored in the Common Data Format (CDF; Goucher et al. 1994) used by the particles and fields communities.

Most Solar Orbiter science goals are based on coordinated analysis between multiple instruments, and depend on interoperability between these two quite distinct scientific communities.
It is therefore crucial for the scientific outcome of the mission, and for the whole scientific community, to make the full range of Solar Orbiter data easily accessible and interpretable by defining data products that are consistently formatted in a transparent and standard manner. To this end, standard metadata keywords have been agreed upon for both FITS and CDF data files produced by Solar Orbiter (MADAWG 2018).

The Solar Orbiter metadata standard for FITS files follows the FITS standard, combined with the World Coordinate System (WCS) standard for coordinates defined in a series of papers (Greisen \& Calabretta 2002; Calabretta \& Greisen 2002; Greisen et al. 2006; Rots et al. 2015), and adapted for solar coordinates (Thompson 2006). Where possible, keywords already in common use by other missions (STEREO, PROBA2 (PRoject for On-Board Autonomy), Hinode, IRIS (Interface Region Imaging Spectrograph), etc.) were adopted to maximise cross-mission compatibility, and enable the use of Solar Orbiter data in already existing tools such as (J)Helioviewer (Müller et al. 2017). In some cases, the evolving FITS standard necessitated a deviation from common practice. For example, the keywords XPOSURE and TELAPSE were adopted in place of the older EXPTIME keyword to be compatible with the WCS time standard (Rots et al. 2015). Not all keywords in the Solar Orbiter metadata standard are required to appear in every FITS header, as some are only appropriate for certain kinds of data. However, when these keywords are used, they must conform to the definition in the metadata standard. A complete list of adopted keywords is given in Table 6. Along with basic FITS files, the metadata standard also includes support for IMAGE and binary table (BINTABLE) extensions.

A function of the Solar Orbiter metadata standard is to document the context in which the data were taken. This includes standardised keywords regarding the instrument configuration during the observation, such as FILTER for the name of any bandpass filter used, or NSUMEXP for the number of exposures summed together onboard to form the observation (in this latter case, the keyword XPOSURE is the total effective exposure time of all the exposures added together). The SOOP associated with the observation is identified through the keywords SOOPNAME, SOOPTYPE, OBS_ID which gives the ID number of the SOOP, and TARGET which identifies the SOOP target (e.g. "AR", "QS", "limb", etc.). We note that the keyword OBJECT has a similar definition, but is tied to the instrument configuration at the time, and not to the SOOP.

Use of the WCS formalism allows the data to be completely specified in terms of both pointing and observation time, together with the units for both the data themselves and the coordinates in which they are specified. Other keywords specify the position of the S/C at the time of the observation in a variety of coordinate systems (Thompson 2006). For example, the keywords CRLN_OBS, CRLT_OBS, DSUN_OBS can be used to express the position of the $\mathrm{S} / \mathrm{C}$ in Carrington Heliographic longitude and latitude, along with the distance from Sun centre, while HCIX_OBS, HCIY_OBS, HCIZ_OBS express the $x, y, z$ Heliocentric Inertial (HCI) coordinates. The $\mathrm{S} / \mathrm{C}$ velocity can be expressed with the keywords HCIX_VOB, HCIY_VOB, HCIZ_VOB, with HCI explicitly chosen as it is an inertial system. The WCS formalism allows for alternative coordinate systems to be defined in the header along with the primary coordinate system. For example, SoloHI may choose to add a celestial coordinate system to characterise the locations of stars and planets visible in their FOV.

The information contained in Solar Orbiter FITS headers depends on the data level. In general, complete metadata, including S/C attitude, are only available beginning with Level 2 
Table 5. Low-latency data products provided by the Remote-Sensing payload.

\begin{tabular}{|c|c|c|}
\hline Instr. & Type of data product [use case(s)] & Cadence \\
\hline \multirow[t]{3}{*}{ EUI } & Beacon data: low-resolution FSI images (174/304 ̊) $[2,3]$ & $30 \mathrm{~min}$ \\
\hline & Synoptic data: low-cadence, high-quality FSI images [1] & 1 set/day \\
\hline & If applicable: sample EUI/HRI 174 \& EUI/HRI $\mathrm{Ly}_{\mathrm{L} \alpha}$ data $[1,3]$ & $1 \mathrm{set} / \mathrm{day}$ \\
\hline \multirow[t]{2}{*}{ PHI } & Precursor and quicklook data: $1024 \times 1024$ pixels continuum image $\&$ LOS-magnetogram [all*] & 1 set/day ${ }^{(* *)}$ \\
\hline & Calibration: snapshot of calibration products [1] & Start/end RSW \\
\hline SPICE & Each science study is preceded by LL version with same scientific performance [1] & Varying \\
\hline \multirow[t]{2}{*}{ SoloHI } & Regular set of horizontal strips to build "J-maps" [3] & TBD \\
\hline & Compressed sample of detectors or regions of interest [1] & \\
\hline \multirow[t]{2}{*}{ Metis } & 2 VL images for total brightness +1 UV image (all rebinned) [1] & 1 set/day \\
\hline & 8 light curves, one for each VL sector & \\
\hline \multirow[t]{3}{*}{ STIX } & Light curves per energy band [all $\left.{ }^{(*)}\right]$ & $4 \mathrm{~s}$ \\
\hline & Flare information data [all $\left.{ }^{(*)}\right]$ & $8 \mathrm{~s}$ \\
\hline & Energy calibration spectra [1] & \\
\hline
\end{tabular}

Notes. The use case(s) that each of the data sets fulfill (Sect. 6) are marked between brackets. ${ }^{(*)} \mathrm{PHI}$ and STIX implement data selection onboard but only from an internal buffer, not from SSMM. ${ }^{(*)} \mathrm{PHI}$ will usually provide full-disc data; in case PHI/FDT is not in operation for more than one day; PHI/HRT data will be provided instead.

Table 6. Agreed FITS keywords.

\begin{tabular}{|c|}
\hline Basic \\
\hline $\begin{array}{l}\text { SIMPLE, BITPIX, NAXIS, NAXIS } i \text {, EXTEND, } \\
\text { LONGSTRN, BSCALE, BZERO, BTYPE, BUNIT, } \\
\text { DATAMIN, DATAMAX, BLANK, COMMENT, CHECKSUM, } \\
\text { DATASUM, HISTORY, XTENSION, PCOUNT, GCOUNT, } \\
\text { EXTNAME, TFIELDS, TFORM } i \text {, TTYPE } i \text {, TUNIT } i \text {, } \\
\text { TDIM } i \text {, END }\end{array}$ \\
\hline General description \\
\hline $\begin{array}{l}\text { FILENAME, FILE_RAW, PARENT, APID, DATE, } \\
\text { DATE-OBS, DATE-BEG, DATE-AVG, DATE-END, } \\
\text { TIMESYS, TIMRDER, TIMSYER, OBT_BEG, } \\
\text { OBT_END, LEVEL, ORIGIN, VERS_CAL, VERSION, } \\
\text { SOOPNAME, SOOPTYPE, OBS_ID, TARGET }\end{array}$ \\
\hline Instrument and observation configuration \\
\hline $\begin{array}{l}\text { OBSRVTRY, TELESCOP, INSTRUME, DETECTOR, } \\
\text { OBJECT, OBS_MODE, STUDY_ID, FILTER, } \\
\text { WAVELNTH, WAVEMIN, WAVEMAX, WAVEBAND, } \\
\text { XPOSURE, NSUMEXP, TELAPSE, TRIGGERD, PXBEGn, } \\
\text { PXENDn, NBIN } i, \text { NBIN, COMPRESS, COMPQUAL }\end{array}$ \\
\hline WCS attitude parameters \\
\hline $\begin{array}{l}\text { WCSAXES, WCSNAME, CTYPE } i, \text { CUNIT } i, \text { PC } j \_i \text {, } \\
\text { CDELT } i \text {, CROTA, CRVAL } i, \text { CRPIX } i \text {, CRDER } i \text {, } \\
\text { CSYER } i \text {, LONPOLE, SPECSYS, VELOSYS }\end{array}$ \\
\hline Solar ephemeris data \\
\hline $\begin{array}{l}\text { RSUN_ARC, RSUN_REF, SOLAR_BQ, SOLAR_PQ, } \\
\text { SOLAR_EP, CAR_ROT, HGLT_OBS, HGLN_OBS, } \\
\text { CRLT_OBS, CRLN_OBS, DSUN_OBS, HEEs_OBS, } \\
\text { HCI } s \_O B S, \text { HCI } s \text { _VOB, HAEs_OBS, HEQ } s \_O B S, \\
\text { GSEs_OBS, OBS_RV, EAR_TDEL, SUN_TIME, } \\
\text { DATE_EAR, DATE_SUN }\end{array}$ \\
\hline
\end{tabular}

Notes. The parameters $i$ and $j$ refer to axis indices, and $s$ can take the values $\mathrm{X}, \mathrm{Y}$, or Z. Some keywords also have variations for binary tables. Complete definitions, including units, are given in MADAWG (2018).

(L2). Low-latency files (LL01, LL02, etc.) used for operations planning differ from their normal science quality equivalents in that only preliminary calibrations have been applied. During times of low downlink performance, low-latency data may be the only data available for several months. In this case instrument teams may choose to produce an enhanced version of the lowlatency data with better calibration and more complete metadata in the header, which would then qualify as L2 data or above.

\section{Summary}

The RS instrument package of Solar Orbiter will provide us with a new view of our Sun, from its interior out to the solar wind. Some of their measurements will be unprecedented, like the highest-ever resolution UV and EUV images, or observations of the Sun's polar regions, including the direct Doppler velocity of the fast solar wind. The development of instruments that are prepared to face 13 solar constants has been a challenge that could only be met by intelligent design and the dedication of the teams. The coordination effort led by the RSWG contributed to the optimisation of the instruments' performance and of the joint operation schemes in the context of the overall mission objectives.

Acknowledgements. A. V. is supported by NRL grant N00173-16-1-G029. D. M. L. is grateful to the Science Technology and Facilities Council for the award of an Ernest Rutherford Fellowship (ST/R003246/1).

\section{References}

Antonucci, E., Romoli, M., Andretta, V., et al. 2020, A\&A, 642, A10 (Solar Orbiter SI)

Auchère, F., \& Artzner, G. E. 2004, Sol. Phys., 219, 217

Auchère, F., Boulade, S., Koutchmy, S., et al. 1998, A\&A, 336, L57

Auchère, F., DeForest, C. E., \& Artzner, G. 2000, ApJ, 529, L115

Bloomfield, D. S., Gallagher, P. T., Marquette, W. H., Milligan, R. O., \& Canfield, R. C. 2016, Sol. Phys., 291, 411

Bonte, K., Berghmans, D., De Groof, A., Steed, K., \& Poedts, S. 2013, Sol. Phys., 286, 185

Calabretta, M. R., \& Greisen, E. W. 2002, A\&A, 395, 1077

Couvidat, S., Schou, J., Hoeksema, J. T., et al. 2016, Sol. Phys., 291, 1887

Defise, J. M., Clette, F., \& Auchère, F. 1999, in EUV, X-Ray, and Gamma-Ray Instrumentation for Astronomy X, eds. O. H. Siegmund, \& K. A. Flanagan, Proc. SPIE, 3765, 341

Fox, N. J., Velli, M. C., Bale, S. D., et al. 2016, Space Sci. Rev., 204, 7

García-Marirrodriga, C., Pacros, A., Strandmoe, S., et al. 2020, A\&A, in press, https://doi.org/10.1051/0004-6361/202038519 (Solar Orbiter SI) 
Gordino, M., Auchère, F., \& Bocchialini, K. 2020, A\&A, submitted

Goucher, G., Love, J., \& Leckner, H. 1994, in Solar-terrestrial Energy Program. The Initial Results from STEP Facilities and Theory Campaigns, Proceedings of the 1992 STEP Symposium/5th COSPAR Colloquium, 691

Greisen, E. W., \& Calabretta, M. R. 2002, A\&A, 395, 1061

Greisen, E. W., Calabretta, M. R., Valdes, F. G., \& Allen, S. L. 2006, A\&A, 446 747

Howard, R. A., Moses, J. D., Vourlidas, A., et al. 2008, Space Sci. Rev., 136, 67

Howard, R. A., Vourlidas, A., Colaninno, R. C., et al. 2020, A\&A, 642, A13 (Solar Orbiter SI)

Kaiser, M. L., Kucera, T. A., Davila, J. M., et al. 2008, Space Sci. Rev., 136, 5

Kosugi, T., Matsuzaki, K., Sakao, T., et al. 2007, Sol. Phys., 243, 3

Krucker, S., Hurford, G. J., Grimm, O., et al. 2020, A\&A, 642, A15 (Solar Orbiter SI)

MADAWG 2018, Metadata Definition for Solar Orbiter Science Data, Solar Orbiter technical note SOL-SGS-TN-0009 Iss 2.3, ESA/ESAC

Mihalas, D., \& Binney, J. 1981, Galactic Astronomy: Structure and Kinematics, 2nd edn. (San Francisco: W.H. Freeman and Company)

Morales, C., Trapero, J., Gómez, J. F., et al. 2000, ApJ, 530, 403

Müller, D., Marsden, R. G., St. Cyr, O. C., Gilbert, H. R., \& The Solar Orbiter Team 2013, Sol. Phys., 285, 25

Müller, D., Nicula, B., Felix, S., et al. 2017, A\&A, 606, A10

Müller, D., St. Cyr, O. C., Zouganelis, I., et al. 2020, A\&A, 642, A1 (Solar Orbiter SI)

Owen, C. J., Bruno, R., Livi, S., et al. 2020, A\&A, 642, A16 (Solar Orbiter SI)

Pence, W. D., Chiappetti, L., Page, C. G., Shaw, R. A., \& Stobie, E. 2010, A\&A 524, A42

Pesnell, W. D., Thompson, B. J., \& Chamberlin, P. C. 2012, Sol. Phys., 275,

Rochus, P., Auchère, F., Berghmans, D., et al. 2020, A\&A, 642, A8 (Solar Orbiter SI)

Rots, A. H., Bunclark, P. S., Calabretta, M. R., et al. 2015, A\&A, 574, A36

Rouillard, A. P., Pinto, R. F., Vourlidas, A., et al. 2020, A\&A, 642, A2 (Solar Orbiter SI)

Snow, M., Reberac, A., Quémerais, E., et al. 2013, in A New Catalog of Ultraviolet Stellar Spectra for Calibration, eds. E. Quémerais, M. Snow, \& R. M. Bonnet, 191

Solanki, S. K., del Toro Iniesta, J. C., Woch, J., et al. 2020, A\&A, 642, A11 (Solar Orbiter SI)

SPICE Consortium (Anderson, M., et al.) 2020, A\&A, 642, A14 (Solar Orbiter $\mathrm{SI})$

Thernisien, A. F., Morrill, J. S., Howard, R. A., \& Wang, D. 2006, Sol. Phys., 233,155

Thompson, W. T. 2006, A\&A, 449, 791

Tian, H., Curdt, W., Marsch, E., \& Schühle, U. 2009, A\&A, 504, 239

Vernazza, J. E., Avrett, E. H., \& Loeser, R. 1981, ApJS, 45, 635

Walsh, A. P., Horbury, T. S., Maksimovic, M., et al. 2020, A\&A, 642, A5 (Solar Orbiter SI)

Watanabe, K., Masuda, S., \& Segawa, T. 2012, Sol. Phys., 279, 317

Zouganelis, I., De Groof, A., Walsh, A. P., et al. 2020, A\&A, 642, A3 (Solar Orbiter SI)

1 Université Paris-Saclay, CNRS, Institut d'Astrophysique Spatiale, 91405 Orsay, France

e-mail: frederic. auchere@ias.u-psud.fr

2 National Institute for Astrophysics (INAF), Astrophysical Observatory of Torino, Via Osservatorio 20, 10025 Pino Torinese, Italy
3 RAL Space, STFC Rutherford Appleton Laboratory, Harwell, Didcot OX11 0QX, UK

${ }^{4}$ Naval Research Laboratory, Space Science Division, Washington, DC 20375, USA

5 University of Applied Sciences and Arts Northwestern Switzerland, 5210 Windisch, Switzerland

${ }^{6}$ European Space Agency, ESTEC, PO Box 299, 2200 Noordwijk, The Netherlands

7 Centre Spatial de Liège, Université de Liège, Av. du Pré-Aily, 4031 Angleur, Belgium

8 Max-Planck-Institut für Sonnensystemforschung, Justus-vonLiebig-Weg 3, 37077 Göttingen, Germany

9 Solar-Terrestrial Centre of Excellence - SIDC, Royal Observatory of Belgium, Ringlaan -3- Av. Circulaire, 1180, Brussels, Belgium

10 Southwest Research Institute, 1050 Walnut Street, Boulder, CO, USA

11 Solar Physics Laboratory, Heliophysics Science Division, NASA Goddard Space Flight Center, Greenbelt, MD 2077, USA

12 European Space Agency (ESAC), Camino Bajo del Castillo, Villanueva de la Cañada, 28692 Madrid, Spain

13 INAF - Astronomical Observatory of Capodimonte, Naples, Italy

14 SSL, UC Berkeley, 7 Gauss Way, Berkeley, CA, USA

15 The Johns Hopkins University Applied Physics Laboratory, Laurel, MD, USA

${ }^{16}$ INAF-Catania Astrophysical Observatory, 95123 Catania, Italy

17 Space Systems Research Corp, Alexandria, VA, USA

18 Instituto de Astrofísica de Andalucía (IAA-CSIC), Apdo. de Correos 3004, 18080 Granada, Spain

19 ADNET Systems, Inc., NASA Goddard Spaceflight Center, Code 671, Greenbelt, MD 20771, USA

20 Dipartimento di Fisica e Astronomia, SASS, Universitá degli Studi di Firenze, Largo E. Fermi 2, 50125 Firenze, Italy

21 UCL-Mullard Space Science Laboratory Holmbury St Mary, Dorking, Surrey RH5 6NT, UK

${ }^{22}$ Physikalisch-Meteorologisches Observatorium Davos, World Radiation Center, 7260 Davos Dorf, Switzerland

23 ESA/ESOC, Robert-Bosch-Str. 5, 64293 Darmstadt, Germany

24 Institut de Recherche en Astrophysique et Planétologie, Université de Toulouse (UPS)/CNRS, Toulouse, France

25 Institute of Theoretical Astrophysics, University of Oslo, PO Box 1029 Blindern, 0315 Oslo, Norway

${ }^{26}$ INAF/Osservatorio Astrofisico di Arcetri, Firenze, Italy

27 National Solar Observatory, 3665 Discovery Drive, Boulder, CO 80303, USA

28 Skobeltsyn Institute of Nuclear Physics, Moscow State University, Moscow, Russia

29 School of Space Research, Kyung Hee University, Yongin, Gyeonggi-Do 446-701, Republic of Korea

30 School of Physics, Trinity College Dublin, Dublin 2, Ireland

31 ASRC Federal Space and Defense, Washington, DC, USA

32 ETH-Zürich, IPA, Hönggerberg campus, Zürich, Switzerland

33 Heliophysics Division, NASA HQ, Washington, DC 20546, USA 